\title{
The Radical Consequences of Realistic Satellite Orbits for the Heating and Implied Merger Histories of Galactic Disks
}

\section{Citation}

Hopkins, Philip F., Lars Hernquist, Thomas J. Cox, Joshua D. Younger, and Gurtina Besla. 2008. "The Radical Consequences of Realistic Satellite Orbits for the Heating and Implied Merger Histories of Galactic Disks." The Astrophysical Journal 688 (2): 757-69. https:// doi.org/10.1086/592087.

\section{Permanent link}

http://nrs.harvard.edu/urn-3:HUL.InstRepos:41381609

\section{Terms of Use}

This article was downloaded from Harvard University's DASH repository, and is made available under the terms and conditions applicable to Open Access Policy Articles, as set forth at http:// nrs.harvard.edu/urn-3:HUL.InstRepos:dash.current.terms-of-use\#OAP

\section{Share Your Story}

The Harvard community has made this article openly available.

Please share how this access benefits you. Submit a story.

\section{Accessibility}


Submitted to ApJ, June 13, 2008

Preprint typeset using LTEX style emulateapj v. 08/22/09

\title{
THE RADICAL CONSEQUENCES OF REALISTIC SATELLITE ORBITS FOR THE HEATING AND IMPLIED MERGER HISTORIES OF GALACTIC DISKS
}

\author{
Philip F. Hopkins ${ }^{1}$, Lars Hernquist ${ }^{1}$, Thomas J. Cox ${ }^{1,2}$, Joshua D. Younger $^{1}$, \& Gurtina Besla ${ }^{1}$ \\ Submitted to ApJ, June 13, 2008
}

\begin{abstract}
Previous models of galactic disk heating in interactions invoke restrictive assumptions not necessarily valid in modern $\Lambda \mathrm{CDM}$ contexts: that satellites are rigid and orbits are circular, with slow decay over many orbital periods from dynamical friction. This leads to a linear scaling of disk heating with satellite mass: disk heights and velocity dispersions scale $\propto M_{\mathrm{sat}} / M_{\text {disk. }}$. In turn, observed disk thicknesses present strong constraints on merger histories: the implication for the Milky Way is that $<5 \%$ of its mass could come from mergers since $z \sim 2$, in conflict with cosmological predictions. More realistically, satellites merge on nearly radial orbits, and once near the disk, resonant interactions efficiently remove angular momentum while tidal stripping removes mass, leading to rapid merger/destruction in a couple of free-fall plunges. Under these conditions the proper heating efficiency is non-linear in mass ratio, $\propto\left(M_{\text {sat }} / M_{\text {disk }}\right)^{2}$. We derive the scaling of disk scale heights and velocity dispersions as a function of mass ratio and disk gas content in this regime, and show that this accurately describes the results of simulations with appropriate "live" halos and disks. Under realistic circumstances, we show that disk heating in minor mergers is suppressed by an order of magnitude relative to the expectations of previous analyses. We show that the Milky Way disk could have experienced $\sim 5-10$ independent 1:10 mass-ratio mergers since $z \sim 2$, in agreement with cosmological models. Because the realistic heating rates are non-linear in mass, the predicted heating is dominated by the more stochastic, rare low mass-ratio mergers, and the existence of populations with little or no thick disk does not require fundamental modifications to the cosmology. This also leads to important differences in the predicted isophotal shapes of bulge-disk systems along the Hubble sequence.
\end{abstract}

Subject headings: galaxies: formation — galaxies: evolution — galaxies: active — galaxies: spiral — cosmology: theory

\section{INTRODUCTION}

In the widely-accepted $\Lambda C D M$ cosmology, it is expected that mergers and interactions between galaxies are crucial to galaxy assembly because structure grows hierarchically (e.g. White \& Rees 1978). On this basis, it has been proposed that spheroids are produced when galaxies merge (making bulges in disks and elliptical galaxies; Toomre \& Toomre 1972; Toomre 1977). Both theory (Ostriker \& Tremaine 1975; Maller et al. 2006; Stewart et al. 2007; Fakhouri \& Ma 2008, and references therein) and observation (e.g. Lin et al. 2004; Woods et al. 2006; Barton et al. 2007; Woods \& Geller 2007 ) indicate that "minor" mergers of mass ratios $\lesssim 1: 10$ are commonplace, and that a large fraction $(\sim 1 / 2)$ of the $\sim L_{*}$ galaxy population has experienced a "major" merger (mass ratio $\lesssim 1: 3$ ) since $z \sim 2-3$ (Bell et al. 2006; Bridge et al. 2007; Kartaltepe et al.2007; Lotz et al.2008; Lin et al. 2008).

The ubiquity of mergers has raised the possibility that cold, thin disks of galaxies may not survive to $z=0$ in hierarchical models. From numerical simulations (e.g. Barnes 1988, 1992; Hernquist 1992, 1993) it has been known for some time that major mergers destroy stellar disks entirely and cause a large fraction of disk gas to lose angular momentum through gravitational torques (e.g. Barnes \& Hernquist 1991, 1996) together yielding dense, quasi-spherical bulges (at least in typical situations such at those at low redshift, where the gas fractions are not large; see e.g. Hopkins et al. 2008). Moreover, although minor mergers (at least those with mass ratios

\footnotetext{
${ }^{1}$ Harvard-Smithsonian Center for Astrophysics, 60 Garden Street, Cambridge, MA 02138

${ }^{2}$ W. M. Keck Postdoctoral Fellow at the Harvard-Smithsonian Center for Astrophysics
}

$\lesssim 10: 1)$ likely do not destroy disks, they are almost an order of magnitude more frequent than major mergers and as such may pose an even more severe problem for disk survival.

In the $\Lambda \mathrm{CDM}$ cosmology it is unlikely than any disk with a significant stellar age has existed $\sim 5-10 \mathrm{Gyr}$ without experiencing a merger of mass ratio 10:1 or larger. Analytic arguments (Ostriker \& Tremaine 1975; Toth \& Ostriker 1992; Sellwood et al. 1998) and early generations of numerical simulations (Quinn 1984; Quinn \& Goodman 1986; Hernquist 1989; Ouinn et al. 1993; Hernquist \& Mihos 1995; Walker et al. 1996; Naab \& Burkert 2003) indicate that gaspoor minor mergers can convert a large fraction of a stellar disk into bulge and dynamically heat the disk. The observed thinness of galactic disks suggests that this may be a severe problem: Toth \& Ostriker (1992) argued that large disks such as that in the Milky Way could not have undergone a merger of mass ratio $\lesssim 10: 1$ in the last $\sim 10 \mathrm{Gyr}$. More recently e.g. Gilmore et al. (2002); Wyse et al. (2006) and Stewart et al. (2007) emphasized that the tension between these constraints and the expectation in CDM models that a number of such mergers should occur implies either a problem with our understanding of hierarchical disk formation or a challenge to the concordance cosmological model.

Given the many successes of the $\Lambda$ CDM model and observations that disks do experience (and survive) a large number of mergers, it seems likely that the main uncertainty is our understanding of disk galaxy evolution. This has focused attention on the problem of forming disks in a cosmological context. A number of studies have argued that detailed modeling of gas physics, star formation, and feedback, along with high numerical resolution, are required to simulate 
disk formation and understand how disks survive their violent merger histories (Weil et al. 1998; Sommer-Larsen et al. 1999, 2003; Thacker \& Couchman 2000, 2001; Abadi et al. 2003; Governato et al. 2004, 2007; Robertson et al. 2004; Okamoto et al. 2005; Scannapieco et al. 2008). But there has been relatively less focus on whether these simulations produce disks - even where the disks avoid being converted into bulges - that are sufficiently thin relative to observational constraints. Interestingly, more recent attempts to model disk heating in a cosmological context (see e.g. Benson et al. 2004; Kazantzidis et al. 2007) suggest that disk heating may be significantly less efficient than has been assumed.

Indeed, a closer examination of this question reveals that much of the conventional wisdom and the implied constraints on the merger history of massive disks (and the Milky Way in particular) is fundamentally premised on historical assumptions that do not apply given what we now know about the $\Lambda \mathrm{CDM}$ framework. For example, implicit in the analysis of Toth \& Ostriker (1992) and others is the assumption that the orbits of merging satellites can be described as being nearly circular, slowly spiraling in owing to dynamical friction, while the satellite remains relatively intact. Early simulations often adopted these choices - modeling the satellites as rigid point masses on almost perfectly circular orbits, including relatively low-mass and smooth dark matter halos (such that the background density is low, and the in-spiral of the satellite therefore relatively slow and without much tidal stripping), and/or using rigid (as opposed to full N-body or "live") background disk/bulge/halo potentials. Moreover, these calculations were almost always dissipationless, and did not allow for the role of gas in making the system more robust against mergers and heating. Under these conditions, it is straightforward to show that the disk heating owing to a merger is linear in the mass of the satellite. This fundamentally underlies the constraint above: because the scaling is linear, it is possible to transform an observed disk thickness or velocity dispersion into an integral constraint on the allowed merger history of a galaxy.

Given cosmological constraints and numerical capabilities at the time, these were reasonable assumptions. However, with improvements in simulations, yielding the ability to perform true cosmological simulations with resolved subhalo populations, and better understanding of cosmological parameters and estimates of halo masses and sizes, it is clear that the assumptions underlying these early works are not appropriate. For example, it is now well-established that realistic cosmological orbits are quite radial, not circular. This is true on all mass scales, from large dark matter substructures which populate halos (van den Bosch et al. 1999; Benson 2005; Khochfar \& Burkert 2006) to unresolved background velocity flows in the diffuse stellar and dark matter halo (Cole \& Lacey 1996; Abadi et al. 2006). Given that realistic galaxy halos exhibit considerable substructure with large subhalo populations, it is generally true that systems which pass near a disk - those of interest for mergers and disk heating - will be those which can transfer angular momentum and collide with the disk on highly radial passages, rather than circular in-spirals (Font et al. 2001; Benson et al. 2004).

Moreover, properly accounting for live halos and disks leads to qualitatively different angular momentum transfer in the final stages of galaxy mergers: once a satellite reaches a radius comparable to the disk, resonances between the orbital motion and internal motions of the disk and halo remove angular momentum efficiently and lead to a rapid, radial coa- lescence in a couple of dynamical times (rather than the large number of orbital times required for a dynamical friction inspiral in the case of mass ratios $\gtrsim 1: 10)$. Furthermore, allowing halos to have the large masses expected in modern cosmologies and resolving disks with $\mathrm{N}$-body approaches, with proper live secondaries, leads to efficient tidal stripping of the satellite once it begins to pass through a disk, transferring orbital energy to the central bulge and extended dark matter halo (preferentially the hottest components of the system, rather than the disk).

The historical picture of "sinking" satellites on slowly decaying, circular orbits is based in part on an old view of the orbits of the Large and Small Magellanic Clouds (LMC and SMC). Recent observations, however, indicate that the LMC and SMC are on a high-velocity passage near pericenter and are only marginally bound to the Milky Way (Kallivayalil et al. 2006a.b; Piatek et al. 2008). Besla et al. (2007) used these results and modern cosmological models of the Milky Way halo to infer their orbital motion, and argued that they are in fact on highly eccentric radial orbits and are just now executing their first passage. Radial orbits are not uncommon in the local group: Howley et al. (2008) recently demonstrated that NGC 205 (an M31 dwarf) is also likely on a radial passage near its escape velocity; Leo I is similar (Mateo et al. 1998; Sohn et al. 2007; Muñoz et al. 2008). This, and the arguments above, suggest that constraints regarding the merger history of the Milky Way and disk heating should be revisited in light of modern cosmological expectations.

One might think that the difference between the conventional lore on satellite heating and allowing for more realistic orbits would amount to a simple numerical correction - i.e. that the differences outlined above can be subsumed into an appropriate correction of the efficiency of disk heating, but will not fundamentally alter the scaling laws derived in the past (namely that disk heating is linear in merger mass). In what follows, we show that this is not the case. At a basic level, the linear scaling resulting from earlier assumptions is pathological, and allowing for moderate deviations in line with what is expected in a $\Lambda \mathrm{CDM}$ cosmology yields a qualitatively different scaling and a non-linear dependence of disk heating on satellite mass. This implies more than an order-ofmagnitude suppression of disk heating in intermediate massratio minor mergers, and essentially no contribution to disk heating from very minor mergers (unlike if the heating were linear, in which case there would be equal contributions to disk thickness from all logarithmic intervals in satellite and subhalo masses). We show that the scaling we derive, nonlinear (second-order) in the satellite mass, is dynamically inevitable and borne out in numerical simulations.

We outline the physics of interest in $\S 2$, explaining why cosmologically realistic merging orbits (even when nearly circular initially) tend to be highly radial by the time of coalescence and how this differs from the assumption of decaying circular orbits $(\S 2.1)$, demonstrating how this changes the qualitative scaling of disk heating with mass ratio $(\$ 2.2)$, deriving full analytic expressions for the disk thickness and vertical velocity dispersion as a function of merger and disk properties for the radial cases of interest $(\$ 2.3)$, and testing that these equations accurately describe the results of full numerical simulations $(\$ 2.4)$. We discuss the accuracy and robustness of these approximations in $\S 2.5$, examining when they are or are not appropriate. In $\S 3$ we consider the consequences of the different scalings owing to radial versus cir- 
cular orbits, in terms of the allowed merger histories of the Milky Way and other massive disk galaxies ( $\$$ 3.1) and the shapes of galaxies along the Hubble sequence ( $\$$ 3.2). Finally, we summarize our results and discuss some of their cosmological implications and applications to other models and observations in $\S 4$

\section{WHY DO REALISTIC ORBITS MAKE A DIFFERENCE?}

Toth \& Ostriker (1992) developed a simple model for disk heating by a merger with a satellite. They assumed that the satellite - of mass $M_{2}$, with respect to the primary mass $M_{1}-$ was on a circular or nearly circular orbit, which decayed by dynamical friction. Since the energy of an orbit with semimajor axis $a$ is $\sim G M_{1} M_{2} / a$, the orbital energy lost by the satellite (which they assumed was converted into kinetic energy of the nearby stars and dark matter, which themselves are the agents slowing down the orbit) in some annulus as the orbit decays from $a \rightarrow a-\mathrm{d} a$ is $\sim\left(G M_{1} M_{2} / a\right)(\mathrm{d} a / a) \propto M_{2}$. Since the vertical potential for an isothermal sheet $\propto g H$ and the kinetic energy $\propto \sigma_{z}^{2}$, given the assumption that once the disk relaxes it will obey the virial theorem $(E=3 T$, for a planar geometry) this implies that the local scale height $H$ is proportional to the total energy deposited in that annulus ( $H \propto M_{2}$, if the disk was initially cold). In detail, they obtained that at a cylindrical radius $R$ in the disk the average change in scale height owing to a merger is

$$
\frac{\Delta H}{R}=\left(\frac{0.49}{1+0.434 \tilde{H}_{0}}\right)\left(\frac{M_{2}}{M_{1}[<r]}\right),
$$

where $\tilde{H}_{0} \equiv 10 H_{0} / R \sim 1$ is some internal (non-merger induced) scale height, but in any case the dependence on this term is weak; the primary scaling is $\Delta H / R \sim M_{2} / M_{1}$. Indeed, simulations confirm that this scaling does reasonably describe the behavior of the disk in the limit of of a rigid satellite on a circular orbit (see e.g. the discussion in Benson et al. 2004), and that adopting the local virial theorem is a good approximation for deriving the scale height of the disk, given some energy deposition (Ouinn et al. 1993).

\subsection{How Are Real Orbits Different?}

However, a fundamental assumption here, that the orbits are circular and decay slowly (in a gentle circular in-spiral) by dynamical friction while the satellite remains intact, bears little resemblance to realistic orbits in $\Lambda \mathrm{CDM}$ cosmologies. It is now known that satellites merge on highly eccentric, nearly radial orbits (see e.g. van den Bosch et al. 1999; Benson 2005; Khochfar \& Burkert 2006). Moreover, the decay of the orbit, while broadly governed by similar scalings such that e.g. the dynamic friction timescale is not a bad approximation (in an order-of-magnitude sense; see e.g. van den Bosch et al. 1999; Velazquez \& White 1999; Boylan-Kolchin et al. 2008), is usually nothing like the smooth circular decay assumed in Toth \& Ostriker (1992).

Typically, the infalling satellite and primary are deformed after first passage, and resonances between the internal frequencies of the systems and their orbital frequencies allow efficient transfer of angular momentum from the orbit to the halo, while the systems linger near apocenter after the passage. They then fall in on a nearly radial orbit and quickly merge, with the braking of the secondary orbit occurring in a couple of quickly damped radial oscillations through the center of the primary. Even if first passage is at large impact parameter, this process will still generally play out, but perhaps with one or two additional, increasingly radial, passages. At the same time, once the satellite approaches the radii of interest - where it is actually crossing and penetrating the disk, so that disk heating can be effective - it rapidly begins to be tidally stripped and destroyed by the gravitational interaction, and cannot execute a large number of orbits without losing some significant amount of mass (such that only the first couple of passes are the dominant perturbation, not the sum over all passages).

The radial nature of these passages in part owes to cosmologically radial orbits, but it is also an inevitable consequence of any orbits that will actually merge in a dark-matter dominated universe. To see why this is true, consider the nature of dynamical friction for a satellite at some instantaneous threedimensional radius $r$ from the primary center. Technically, the approximation of slow circular orbital decay owing to dynamical friction, which implicitly relies upon the background being infinite, homogeneous, isotropic, and (in a bulk sense) unchanged by the motion and gravity of the secondary, is valid only when the enclosed mass within a sphere of radius $r$ is much greater than the gravitational mass (baryons plus whatever dark matter halo remains bound) of the secondary. So, for example, a small secondary far out in the halo (e.g. the common case of a satellite at large $r$ in a massive cluster) can experience orbital decay owing to traditional dynamical friction.

However, once it reaches a radius where the enclosed mass is somewhat comparable to the satellite mass, this orbital decay approximation is clearly no longer appropriate (consider e.g. the limit of two identical point masses, one the satellite - when they are close enough that the mass of the background halo is negligible, they would just enter into a stable, marginally bound binary orbit, and coalesce slowly). Effects like these are well-known even in numerical simulations that model the idealized case of a rigid satellite in a smooth halo (see e.g. Hashimoto et al. 2003, who argue for a radiallyvarying Coulomb logarithm to match $\mathrm{N}$-body results and the analytic prediction), although these do not include the resonant effects that ultimately remove most of the orbital angular momentum. For a satellite that is small relative to the primary $\left(M_{2} / M_{1} \ll 0.1\right.$, say), this radius may be negligibly small. However, for a satellite that is even a moderate fraction of the primary mass $\left(M_{2} / M_{1} \gtrsim 0.1\right)$, this will happen, for any reasonable mass profile, when the secondary gets near of order the scale length of the primary (considering the halos of the systems, this is really the scale length of the primary halo - i.e. substantially larger even than the scale length of the primary disk). This is precisely the radius of interest for disk heating. In other words, by the time the secondary is close enough to the primary that it can actually move through and heat the stellar disk, it is, by definition (for any secondary which is a significant mass fraction of the primary) in a regime where dynamical friction and circular orbital decay are no longer valid approximations.

Rather, as we noted above, it is in this situation that resonances between the internal velocity of the satellite and its orbital motion will allow efficient transfer of orbital angular momentum, leading to a radial infall. Because these resonances act efficiently when the internal velocities of the galaxies are comparable to their orbital velocities, it is precisely the regime where the secondary is near the primary - where circular orbital decay from dynamical friction ceases to be a valid approximation - when these mechanisms act efficiently. Because internal motions (of at least some parts of the halo) 
are in resonance with those of the orbit, that dark matter is strongly accelerated, removing angular momentum from orbital motion far more efficiently than dynamical friction, allowing an orbit to transform from nearly circular to nearly radial in just one or two passages. Since the angular momentum of the orbit is transferred quickly in a passage (where the system spends most time near some apocenter), the satellite cannot gently spiral in but must return from apocenter in a radial plunge. For a satellite falling in on a radial orbit, it is effectively a free-fall at the escape velocity. The braking by dynamical friction on this infall is weak as the system freefalls (recall, it takes many circular orbits for the orbit to decay by standard dynamical friction).

Rather, when the secondary finally punches through the primary and begins to oscillate through the primary on a radial orbit, violent relaxation (the scattering of stars in the secondary and in the center of the primary) off of the timedependent central potential transfers energy from the radial orbit of the secondary to the motion of the central stellar distribution (preferentially to whatever stars are already "hottest" in the center of the galaxy). This quickly damps out the orbit, completing coalescence in a couple of dynamical times. The energy of the orbit, by this rationale, will largely have gone into heating the bulge of the remnant. This is both because the bulge dominates the stellar mass density at the center of the galaxy, and because the hot/spherical component of the galaxy (having a more isotropic orbit distribution, with a much larger population on radial orbits compared to a cold disk) is in resonance with the orbital decay of the secondary. Some new bulge will be created by violent relaxation and heating of the central stellar populations - we show in Hopkins et al. (2008) that the population violently relaxed is given by the stars within a radius enclosing a stellar mass comparable to the stellar mass of the secondary. In short, a mass and energy $\sim M_{2}$ is added to a classical bulge from the infall, not to heating the disk.

A similar caveat applies to considering a constant-mass secondary. Implicit in the circular orbit in-spiral approximation - in fact, critical to the $\Delta H / R \propto M_{2} / M_{1}$ scaling - is the idea that the satellite heating is integrated over a large number of orbits crossing a given radius $r$ while the orbital angular momentum gradually decays. Clearly, if the satellite is significantly stripped of mass and/or partly destroyed by shocking or tidal interactions after a few orbits, then it is only the first single passage or couple of passages that dominate the heating. In such a case, modulo a geometric prefactor, the effects should be identical to the single radial infall or plunge scenario described above (even if the orbit is still formally perfectly circular). In short, if the satellite loses mass after a couple of passages, then the dominant passages seen by the disk will be the first couple, which will heat the system the same whether they are circular or radial (the exact direction in which the secondary is traveling does not enter into the second-order heating below).

Another way of phrasing this is to note that a significant fraction of the orbital energy is no longer lost to heating the disk, but carried away by the tidally stripped material (which could itself heat the disk in principle, but numerical experiments show that it generally is added to the halo and in more spherical fashion than the disk, while retaining its coherent orbital motion rather than heating nearby material, and moreover because the material is, essentially by requirement in order to be stripped, thin and diffuse relative to the potential nearby, it cannot effect significant heating in the manner of the entire satellite if it moves through the disk; see e.g. Kazantzidis et al. 2007). Moreover, this process can accelerate the orbital angular momentum loss, by shedding angular momentum from the orbit preferentially in the stripped material (there is a general tendency for angular momentum transport to the least-bound material in interactions).

As was the case above for the resonant interactions that remove angular momentum, we can roughly derive the radius where stripping is expected to become important, and find (unsurprisingly) that it is again precisely the radius of interest, i.e. the radius where the secondary begins to actually interact with and cross the primary disk (since the primary disk is much more dense than its extended halo, this will almost always be the case). Even ignoring the more dense baryonic components, if we model the secondary and primary as Hernquist (1990) profile spheres with effective radii $a \propto M^{1 / 3}$ (as expected and observed for both halos and disks; see e.g. Mo et al. 1998; Shen et al. 2003), then calculate the formal tidal radius $\tilde{r}=r_{2} / a_{2}$ within the secondary as a function of the separation $x=r / a_{1}$, the dependence on mass ratio cancels out entirely and we have $\tilde{r}(1+\tilde{r})^{2}=(1+x)^{3} / 2$ - i.e. material within a fraction $\approx 0.8\left(r / a_{1}\right)$ times the effective radius of $M_{2}$ will be stripped when the satellite is at a distance $r$ relative to the effective radius $a_{1}$ of the primary. In short, precisely the time of interest, when the satellite enters the effective radius of the primary, is when the satellite begins to be rapidly stripped (for a more details, see Benson et al. 2004).

\subsection{How Much Heating Arises From Realistic Orbits?}

How much disk heating is effected in this process? There are several ways to cast this problem, but all yield the same behavior. For example, we can even obtain the correct scaling by considering a pure dynamical friction derivation, despite the caveats above, so long as we acknowledge the more realistic nature of the orbits. For simplicity (ignoring temporarily the caveats just given) let us assume that the secondary moves through an isotropic homogeneous background density, with (for analytic convenience, although it makes little difference to the end scaling) an isothermal sphere profile. The instantaneous effective dynamical friction force on the secondary is

$$
\mathbf{F}_{\mathrm{df}}=-0.428 \ln \Lambda \frac{G M_{2}^{2}}{r^{2}} \hat{v},
$$

where $\ln \Lambda$ is the appropriate Coulomb logarithm (we will suppress this for now, but including it is comparable to e.g. the uncertainties owing to details of the profile shape, and numerical experiments suggest $\ln \Lambda \approx 1-2$; see Ouinn \& Goodman 1986; Velazquez \& White 1999; Boylan-Kolchin et al. 2008). The force is, by definition, in the direction of the secondary velocity vector $\hat{v}$ (and opposed to the velocity, hence being negative). If the secondary travels a distance ds, then, an effective work is done by dynamical friction involving a transfer of energy from the orbit of the secondary to the motions of the stars/dark matter that define the background, with magnitude $\mathbf{F}_{\mathrm{df}} \cdot \mathrm{d} \mathbf{s}=\left|\mathbf{F}_{\mathrm{df}}\right||\mathrm{d} \mathbf{s}|$.

If the satellite is on a circular orbit, then this motion $\mathrm{ds}$ is largely in the azimuthal direction, and the radius $r$ decays slowly. The tangential dynamical friction force gives a rate of change of specific angular momentum $L$ of $\mathrm{d} L / \mathrm{d} t=F_{\mathrm{df}} r / M_{2}$. Since for a circular orbit $L=r V_{c}$ (where $V_{c}$ is constant for the isothermal sphere), and $\mathrm{d} s=V_{c} \mathrm{~d} t$, we can rewrite this as $\mathrm{d} r=-0.428\left(G M_{2} / V_{c}^{2} r\right) \mathrm{d} s$, if the satellite remains constant internally throughout the orbit(s). Since the enclosed mass 
within $r, M_{\mathrm{enc}}=V_{c}^{2} r / G$, this is $\mathrm{d} r=-0.428\left(M_{2} / M_{\mathrm{enc}}\right) \mathrm{d} s$. In order for the circular orbit to decay in radius by $\mathrm{d} r$, then, a work $\left(G M_{2} M_{\mathrm{enc}} / r\right)(\mathrm{d} r / r)$ must be done. Since $M_{1} \approx M_{\mathrm{enc}}$, we have just recovered the Toth \& Ostriker (1992) formula, for the specific case of an isothermal sphere. The imparted energy in the annulus $\mathrm{d} r$ is $\propto M_{1} M_{2}$, so the relative heating (i.e. imparted energy relative to the characteristic energy of stars at $r$ in $\left.M_{1}, \sim G M_{1}^{2} / r\right)$ goes linearly in the mass ratio, $\propto M_{2} / M_{1}$. In short, even though the instantaneous force $\propto M_{2}^{2}$ as the secondary orbits, it takes $\sim\left(M_{1} / M_{2}\right) \mathrm{d} r / r$ orbits to decay in radius $\mathrm{d} r$, so the total energy imparted in an annulus scales $\propto M_{1} M_{2}$ (note that this is why it is important that the satellite remain intact in this derivation, since we have implicitly assumed that the same mass $M_{2}$ makes $\sim M_{1} / M_{2}$ crossings of the disk).

The situation is trivial for a radial orbit. Here, $\mathrm{d} s=\mathrm{d} r$, so the work done as the secondary falls inwards by $\mathrm{d} r$ is just $0.428\left(G M_{2}^{2} / r\right)(\mathrm{d} r / r)$. The imparted energy $\propto M_{2}^{2}$, so the relative heating is quadratic in the mass ratio, $\propto\left(M_{2} / M_{1}\right)^{2}$. Note that this is valid in an instantaneous sense (i.e. with $M_{2}$ measured as the system enters the radius $r$ ) even if the system is being stripped or destroyed with each encounter in the disk.

We could instead treat the problem entirely differently, and obtain the same answer. For example, assume instead that we face the opposite regime, where the orbit punches through the disk rapidly instead of being gently dragged, in which case the impact approximation is reasonable. Moreover, this could be used to describe the response of the disk at large $r$ to the damping radial oscillations as the secondary rapidly merges in the center. In either case, a full impact approximation solution (derived following Binney \& Tremaine 1987) follows for circular orbits and a rigid satellite, assuming the impact parameter $b=r$ and impact velocity $V_{i} \sim V_{c}$, and allowing for a number of independent passes $\sim M_{1} / M_{2}$ as required by the energetics for the circular orbit to decay. This yields the same result, that the energy imparted at $r$ goes as $\propto G M_{1} M_{2} / r$. For a radial orbit (or allowing for satellite destruction), we instead assume a penetrating encounter (the radial limit $b=0$ ), with a rapid decay (technically we can fully solve with some exponential radial decay time or stripping/destruction time $\tau \propto t_{\text {dyn }}$, but in any case this only is relevant for the numerical prefactor), and we obtain the same scaling as above, with the energy imparted $\propto G M_{2}^{2} / r$. The details of our derivation only change the exact numerical coefficients.

It is easy to show that these scalings more or less have to be true. For circular orbits and a rigid satellite, as described at the beginning of this section, one can simply take the difference in energy between a circular orbit at radius $r$ and one at radius $r+\mathrm{d} r$, which is $\left(G M_{1} M_{2} / r\right)(\mathrm{d} r / r)-$ this energy must go somewhere, so modulo the degree to which it is spread over the disk (i.e. the Coulomb logarithm), the scaling must be similar. For a radial orbit, there is no difference in orbital energy in a single infall at $r$ and $r+\mathrm{d} r$, and even in the circular case only the first couple of passages matter, so the orbital energy loss is negligible (it takes many circulations for the orbit to decay). The energy exchange must instead come from the deceleration from the wake or interaction of the secondary with the extended primary - i.e. the back-reaction of the surrounding material from the acceleration imparted by $M_{2}$ itself. This effect is necessarily second-order in $M_{2}$ (and arises only because the real distributions of material are extended, rather than point sources). So long as the radial orbit decays quickly once it actually penetrates the galaxy with small impact pa- rameter, there will not be some large number of passages at large $r$ that could change this (and it is almost impossible to arrange a realistic stellar distribution that will not quickly damp a penetrating radial orbit once it passes through the center of the system).

\subsection{A Re-Derivation of Disk Heating for Realistic Orbits}

Here, we present some useful equations for disk heating owing to more realistic orbits. Most of the details are derived in Toth \& Ostriker (1992). The key quantity that differs, depending on the nature of the orbit, is the energy deposition or exchange in an annulus of width $\mathrm{d} r$. It is convenient to express this in terms of the absolute value of the energy exchanged between orbit and background (i.e. the work done $W$, or the energy lost in the orbit) per unit area, $\Delta e=\mathrm{d} W / \mathrm{d} A$. For an isothermal sheet potential (a reasonable approximation in a sufficiently local patch of the disk), the potential (in the vertical direction) is proportional to the scale height $H$ times the local surface gravity and the vertical kinetic energy is just $\mathrm{d} M \sigma_{z}^{2} / 2$. If the system obeys the local virial theorem then $E=T+U=3 T$, and it is straightforward to calculate the heating from some energy input $\Delta e$ :

$$
\begin{aligned}
& H(r)=\frac{2}{3 \pi} \frac{\Delta e}{G \Sigma_{\mathrm{d}}(r)^{2}\left(1+\eta_{h}(r)\right)} \\
& \sigma^{2}(r)=\frac{2}{3} \frac{\Delta e}{\Sigma_{\mathrm{d}}(r)\left(1+\eta_{h}^{\prime}(r)\right)} .
\end{aligned}
$$

Here, $\Sigma_{\mathrm{d}}(r)$ is the disk surface density at $r$ and the term $\left(1+\eta_{h}(r)\right)$ is an order unity (generally small around $R_{\mathrm{d}}$, the scale radius of the disk) correction for the disk-halo gravity. That these are good approximations to full numerical results (e.g. that the system obeys the local virial theorem and that the isothermal sheet approximation is reasonable) has been confirmed in a number of experiments (Quinn et al. 1993; Walker et al. 1996; Velazquez \& White 1999; Benson et al. 2004).

Based on our derivation above, we expect for a radial orbit moving through a background of density $\rho$ that $\Delta e=$ $\left(F_{\mathrm{df}} \mathrm{d} r\right) /(2 \pi r \mathrm{~d} r)$, giving

$$
\Delta e=\alpha \frac{G^{2} M_{2}^{2} \rho}{v^{2}}
$$

in full generality, where $\alpha$ is the appropriate constant depending on the Coulomb logarithm and the isotropy of the background velocity distribution.

Up to this point, we have made no distinction between a dissipationless and dissipational disk. If there is gas in the disk, it can radiate energy efficiently. For simplicity, assume the gas is distributed in the same manner as the stars in the disk with gas fraction $f_{\text {gas }}=M_{\text {gas }} /\left(M_{\text {gas }}+M_{*}\right)$, and that the energy correspondingly couples evenly to the gas and stars (i.e. a fraction $f_{\text {gas }}$ couples to the gas). If the gas can efficiently radiate that energy, then the coupled energy of interest (that which will not just radiate away) is $\Delta e_{\text {eff }}=\left(1-f_{\text {gas }}\right) \Delta e$. Given this effective energy injection, our previous equations for $H(r)$ and $\sigma^{2}$ are unchanged (they will change only on the 2-body relaxation time of the local stellar disk, as energy can be re-exchanged between individual stellar orbits and the thin gas disk, but this timescale is long relative to the heating of interest).

For simplicity, assume the halo is an isothermal sphere, such that the circular velocity is approximately constant as 
a function of radius. This then reduces to

$$
\Delta e=\frac{\alpha}{2 \pi}\left(1-f_{\mathrm{gas}}\right) \frac{G M_{2}^{2}}{r^{3}}
$$

and

$$
\begin{aligned}
& \frac{\Delta H}{R_{e, \text { disk }}}=\alpha_{H}\left(1-f_{\text {gas }}\right)\left(\frac{M_{2}}{M_{\mathrm{d}}}\right)^{2} \tilde{h}\left(R / R_{e, \text { disk }}\right) \\
& \frac{\Delta \sigma_{z}^{2}}{V_{c, \text { disk }}^{2}}=\alpha_{\sigma}\left(1-f_{\text {gas }}\right)\left(\frac{M_{2}}{M_{\mathrm{d}}}\right)^{2} \tilde{s}\left(R / R_{e, \text { disk }}\right)
\end{aligned}
$$

where $\alpha_{H} \approx 1-2$ and $\alpha_{\sigma} \approx 0.3-0.6$ are the appropriate normalization constants and $\tilde{h}\left(R / R_{d}\right) \sim \tilde{s}\left(R / R_{d}\right) \sim 1$ are weak functions of $R / R_{d}$ (for convenience, we define them so that $\tilde{h}(1)=\tilde{s}(1)=1)$.

In detail these quantities will depend on how the orbital decay proceeds as a function of radius. For the precise case derived above on a purely radial orbit that decays instantly at $r \rightarrow 0$ (and isotropic heating), we obtain if the disk is a Mestel (1963) disk (constant circular velocity $V_{c \text {, disk, where }}$ we define $\tilde{v}=\left(V_{c \text {, disk }} / V_{\mathrm{h}}\right)^{2} \approx 1$ relative to the halo circular velocity, and $\left.\Sigma_{\mathrm{d}} \propto 1 / R\right) \alpha_{H}=1.60 \tilde{v}$ and $\tilde{h}(x) \approx[0.7 x+0.3 \tilde{v}]^{-1}$ (giving a roughly constant $\Delta H$ over the radii of interest, similar to what is observed; see e.g. de Grijs \& Peletier 1997; Bizyaev \& Mitronova 2002), with $\alpha_{\sigma}=0.57 \tilde{v}, \tilde{s}(x)=x^{-2}$. For an exponential disk (with $V_{c \text {, disk }}$ defined as the maximum circular velocity of the disk) we obtain $\alpha_{H}=0.68 \tilde{v}, \tilde{h}(x)=$ $\left[2 x^{2} \exp (-x)+0.26 \tilde{v}\right]^{-1}$ and $\alpha_{\sigma}=0.32, \tilde{s}(x)=x^{-2}$.

However, more realistically, the normalization here reflects a sum over various passages as the complex resonant interactions described above rapidly remove angular momentum from the secondary. At the same time, tidal stripping is continuously removing mass, and the gravitational mass of the primary can effectively appear different depending on where in the potential the secondary is located. It is possible to develop a much more complex analytic model that attempts to account for these effects, including e.g. some model of dynamical friction, stripping, disk-satellite shocking, and then time-integrating these equations to produce the numerical constant desired (see e.g. Benson et al. 2004). Although these offer some improvement, it is still unfortunately not possible to follow the proper sources of angular momentum loss (resonant coupling in the orbit) and back-reaction of the halo and secondary in fully analytic form, and because of this, these more sophisticated treatments do not yield a major gain over our much simpler scalings above.

Fortunately, testing these predictions in full numerical simulations (see $\S 2.4$ below) - including live halos and secondaries, systems with a huge number of very small satellites, and gas-rich disks evolved in full hydrodynamics - we find that the scalings above nevertheless accurately describe the disk response, with a simulation-to-simulation scatter of only a factor $\sim 2-3$. Calibrating the normalization of the efficiencies properly to $\mathrm{N}$-body results, we obtain $\alpha_{H} \approx 1.0-2.0$ and $\alpha_{\sigma} \approx 0.3-0.6$, similar to the coefficients estimated analytically. We find that these calibrations are robust across a wide range of simulations and mass ratios, and describe the behavior of $\Delta H / R$ and $\Delta \sigma^{2} / v_{c}^{2}$ well from $\sim 1-5 R_{d}$ (outside of which the disk is fairly ill-defined, both numerically and physically, so we are not surprised that the scalings do not trivially extrapolate to infinite $R$ ).

\subsection{Numerical Tests}

In order to test how robust these scalings are, we compare them with $\mathrm{N}$-body simulations. We compile results from a number of different systematic studies of mergers, in order to see whether the particular choices in a given study are pathological.

Velazquez \& White (1999) consider collisionless mergers of an N-body disk+bulge+halo Milky Way-like model primary (although the system is collisionless, and so can be freely rescaled to arbitrary mass) with N-body King (1966)model (truncated isothermal sphere) secondary satellites of varying concentration and mass ratios 1:5 and 1:10. The authors perform a survey of orbital inclination (from prograde $\theta=0^{\circ}$ to retrograde $\theta=180^{\circ}$ ) and impact parameter (equivalent to varying the initial eccentricity or circularity of the orbit, with $\epsilon_{J} \equiv J / J_{C}(E)$ being the ratio of the orbital angular momentum $J$ to that of a circular orbit $J_{C}$ varied).

Villalobos \& Helmi (2008) consider dissipationless mergers of a $z=0$ Milky Way-like primary and a scaled $z=1$ analog, with disk and satellite properties scaled according to theoretical expectations following Mo et al. (1998) (note that this may in fact over-estimate the redshift corrections, making their $z=1$ case more appropriate for $z \sim 2-3$; see Somerville et al. 2008). They model the primary halo as adiabatically contracted, and merge it with either bulge+halo or disk+halo satellite models of mass ratio 1:5, on varying orbits with a moderately radial impact parameter.

Younger et al. (2008) consider full hydrodynamic merger simulations of two bulge+disk+halo systems, with mass ratios $1: 8,1: 4,1: 3$, and 1:2. In each case, the orbital inclination is surveyed, and two sets of models are run: one with a relatively low gas fraction $\sim 0.2$ in the disk at the time of the merger (corresponding to Milky Way like disks over the last few Gyr) and one with intermediate gas fractions $\sim 0.4$ (corresponding better to intermediate redshift $z \gtrsim 1$ cases). Most of the orbits are parabolic and radial $\left(b \sim 1-2 R_{d}\right)$, but the authors consider limited surveys in the impact parameter and corresponding circularity/initial angular momentum of the orbit (from $b=0-10 R_{d}$ ). Because, for dissipational systems, the gas properties set an absolute units scale, the physical mass and size scale of the galaxies cannot be freely rescaled: the authors examine primarily a Milky Way-like primary, but also consider a limited mass survey spanning a factor $\sim 10$ in lower primary masses.

Hayashi \& Chiba (2006) investigate dissipationless mergers of a disk+bulge+halo primary (including both rigid halos+bulges and restricted halos free to move but not realized in N-body fashion, with an N-body disk; note that Velazquez \& White (1999) argue that these restricted halo models may artificially inflate the resulting scale heights by a factor $\sim 2$, but this is comparable to the scatter in any case). The halo is merged with a large number of satellites (subhalos), drawn in Monte Carlo fashion from a mass spectrum with power-law index $d N / d M \propto M^{-2}$, similar to the sub-Milky Way end of the halo mass function (see also Ardi et al. 2003, who perform a similar exercise and reach similar conclusions). A mass fraction $=0.1$ of the primary is placed into satellites according to that mass distribution, with a their spatial distribution varied systematically (following a Hernquist (1990) profile), and their initial velocity distribution determined assuming they follow the local halo velocity ellipsoid with either an isotropic dispersion tensor $\left(\beta=1-0.5\left(\sigma_{\theta}^{2}+\sigma_{\phi}^{2}\right) / \sigma_{r}^{2}=0\right)$ or a radially anisotropic disper- 

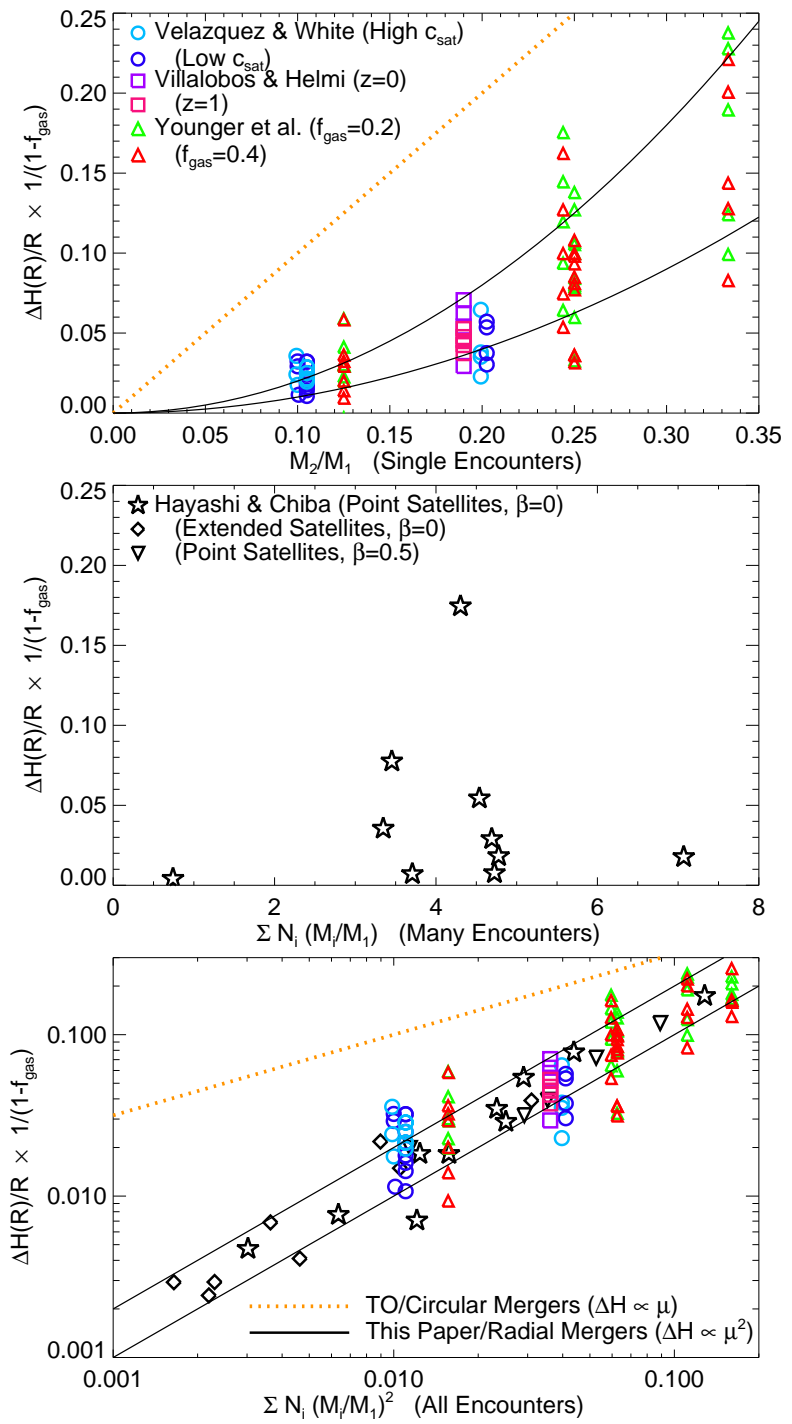

FIG. 1.- Analytic predictions for disk heating in mergers and results of numerical experiments. Top: Disk heating (change in scale height at $\left.R \approx R_{e}\right) \Delta H(R) / R$ versus merger mass ratio $M_{2} / M_{1}$. Contrast our prediction $\Delta H \propto\left(M_{2} / M_{1}\right)^{2}$ (Equation 7); black lines show a factor 2 scatter) and that of Toth \& Ostriker (1992) for rigid satellites on circular orbits ( $\Delta H \propto M_{2} / M_{1}$; orange dotted). We compare N-body simulations of disksatellite mergers of various masses (labeled). Each simulation set surveys orbital parameters (inclination, impact parameter, angular momentum). The dissipationless Velazquez \& White (1999) simulations also survey satellite mass profiles and concentrations (c); the dissipationless Villalobos \& Helmi (2008) simulations scale systems to $z=0$ and $z=1$, and consider both disk and spheroid satellites. The hydrodynamic Younger et al. (2008) simulations survey disk gas content (disk heating scales $\propto\left(1-f_{\text {gas }}\right)$; to factor out this dependence, we plot these points as $\left.\Delta H / R \times 1 /\left(1-f_{\text {gas }}\right)\right)$. Middle: Heating versus the linear sum over encounters in Hayashi \& Chiba (2006) simulations. They consider simultaneous mergers with many $(\sim 100-1000)$ small satellites $\left(M_{2} / M_{1} \sim 0.001-0.1\right)$. Each point is the net heating, integrated over all encounters, for different initial configurations. If heating were linear in $M_{2} / M_{1}$, there should be a linear correlation between the net heating and the linear sum over mergers, $\Sigma N_{i}\left(M_{i} / M_{2}\right)$. Bottom: Heating versus the quadratic sum over all encounters, as predicted by our Equation (7). Numerical experiments confirm our predicted scaling with factor $\sim 2-3$ scatter, and demonstrate that the scaling of disk heating is second-order in $M_{2} / M_{1}$.

sion $(\beta=0.5)$. In addition to their spatial and orbital velocity distributions, the absolute number of subhalos and maximum and minimum masses sampled are varied, and both pointmass subhalos and extended-mass (N-body) subhalo models are considered.
Figure 1 plots the results of these experiments. Specifically, we compile (for the experiments described) the resulting scale height of the disk after the merger, at some radii $\sim 1-4 R_{d}$ (i.e. within a factor of 2 of $R_{e}$, where we do not expect a strong dependence of $H$ on $R$ ). In all cases, the authors evolve corresponding isolated disks, so the value we consider is $\Delta H$, the change in scale height relative to an isolated disk (i.e. subtracting the effects of internal heating). We convert all the results to a uniform definition in order to compare them in a fair manner: we define $\Delta H$ precisely as the median scale-height of stars in the annulus $\mathrm{d} R$ (i.e. exactly half the disk stars in the cylindrical radial annulus $\mathrm{d} R$ should lie in $z= \pm H$ ), and obtain $\Delta H$ as close to the disk half-mass radius $R_{e}$ as possible (but in any case rescale all the results to $\Delta H(R) / R$ for the $R$ measured).

First, we consider the simulations from Velazquez \& White (1999), Villalobos \& Helmi (2008), and Younger et al. (2008), who consider idealized single encounters (as opposed to large numbers of multiple or simultaneous encounters). We compare $\Delta H(R) / R$ as a function of the mass ratio of the encounter $\mu=M_{2} / M_{1}$, for the various simulations, to the analytic predicted scalings from Toth \& Ostriker (1992) (linear in $\mu$ ) and our derivation herein (second-order in $\mu$ ). For the Younger et al. (2008) hydrodynamic simulations, we confirm the predicted scaling $\Delta H \propto\left(1-f_{\text {gas }}\right)$ (and a more limited subset shows that this applies reasonably well over a systematic study varying $f_{\text {gas }}=0-1$; see e.g. Hopkins et al. 2008). To compare uniformly with the other (dissipationless, $f_{\text {gas }}=0$ ) numerical simulations here, we actually plot $\Delta H / R \times 1 /\left(1-f_{\text {gas }}\right)$, so that all simulations are on the same footing. For each set of simulations and mass ratio, there is a factor $\sim 2-3$ scatter owing to a combination of orbital parameters (both orbital inclination and impact parameter) and the detailed choices for the structural properties of the galaxies (recall, the simulations plotted make various and in some cases quite different assumptions about the shapes of secondary galaxies and their mass profiles). In any case, the scatter is still reasonably small, and despite the fact that these simulations include e.g. the more complex realistic orbital structure, tidal stripping, and other effects, they agree quite well with our predicted scalings for the range in normalizations $\alpha_{H} \approx 1-2$.

If we were to consider arbitrary normalizations $\alpha_{H}$ (i.e. ignore the analytic expectations entirely), then for any single encounter, there is of course a formal degeneracy between the dependence of resulting scale height on mass ratio and the efficiency (implicit in $\alpha_{H}$ ). Even for the set of simulations just described, the dynamic range in $\mu$ is relatively small and scatter significant, so if we freely renormalized the Toth \& Ostriker (1992) prediction, we could find a solution that was not a particularly bad match to the numerical results (albeit still not as good a fit as the predicted quadratic dependence, even with a fitted $\alpha_{H}$ that differs from the analytic expectation by almost an order of magnitude). The simulations of Hayashi \& Chiba (2006), however, include the effects of a large number of encounters with a wide range of mass ratios $(\sim 2$ orders of magnitude in $\mu$ ), and can strictly break this degeneracy.

If the true scaling of $\Delta H / R$ were linear in $\mu$, regardless of its normalization, then, in a sum of $N_{i}$ encounters with various systems of mass ratios $\mu_{i}$, the net heating should be linear in the sum of these individual events (modulo some scatter), i.e. $\Delta H / R \propto \Sigma N_{i} \mu_{i}$. Hayashi \& Chiba (2006) consider this, and we show their results (renormalized appropriately 
for our definitions) in Figure 1 Note that because some of the orbits they consider are very long axis and/or energetic, a given satellite (especially in the cases where they use point mass satellites, since there is no stripping/destruction of the satellite) can make many well-separated passes through the disk. The appropriate sum is then over each encounter, treating them separately with $\mu_{i}$ appropriate for the mass ratio at each encounter that penetrates the disk (for this reason, with some satellites making many passages, the sum $\Sigma N_{i} \mu_{i}$ can be $>1$, despite the fact that the total subhalo mass in these simulations is only a fraction of the primary mass). Each point in the figure represents the sum over many small mass ratio encounters with one of the initial configurations described above, typically mergers with $\sim 1000$ satellites spanning a mass spectrum from $\mu_{i} \sim 10^{-3}-10^{-1}$. In any case, the result is essentially a scatter plot, with no significant correlation visible.

If, instead, the appropriate scaling goes as $\mu^{2}$, as we have predicted, then the net heating in this case should behave as $\propto \sum N_{i} \mu_{i}^{2}$. Considering the results from Hayashi \& Chiba (2006) in this comparison, we do indeed find a tight linear correlation (as the authors themselves note). In short, the heating from each of the many encounters $\mu_{i}$ is clearly adding quadratically - i.e. the effective heating per event scales $\propto \mu_{i}^{2}$, not linearly. Because this is a mix of mass ratios, projecting versus $\Sigma N_{i} \mu_{i}$ degrades the correlation increasingly the more the merger history is varied. We also reproduce the simpler merger history simulations from Velazquez \& White (1999), Villalobos \& Helmi (2008), and Younger et al. (2008) in this plot, with our analytic expectations. For these single encounters, we take $N_{i}=1$ with $\mu_{i}$ being the initial mass ratio, appropriate for the fairly idealized mergers these represent (although they vary other properties not considered in the Hayashi \& Chiba (2006) simulations), a reasonable approximation also for the reasons below.

\subsection{How Robust Is This Scaling?}

Given the complexity of the full time-dependent behavior in these simulations, and the wide variation in the physics included and assumptions made by the different authors, it is remarkable that the agreement is as good as it is in Figure 1. Why should our simple scalings (Equations 7, 8) describe the full N-body results as well as they do, with only a scatter of a factor $\sim 2-3$ in $\Delta H / R$ and no dramatic systematic offset between different simulations? There are in fact several reasons for this.

Most important, despite the fact that there is considerable complexity in the orbital decay of satellites, is that once it begins to experience close passages and penetrating encounters with the disk, the satellite is efficiently tidally stripped and destroyed within a couple of passages (see e.g. Ouinn et al. 1993; Velazquez \& White 1999; Benson et al. 2004). The result is that the approximation of a single "plunge" with instantaneous decay/destruction is quite good. Furthermore, the tendency of resonant interactions between disk, halo, and substructure to efficiently remove angular momentum also serves to make final passages more radial, removing some of the dependence that might otherwise result from initially different orbital configurations. Critically, all of these modern simulations include live disks and massive dark matter halos. In addition, differences between the effects of individual passages are averaged out in realistic cosmological ensembles with many encounters.
These numerical experiments also allow us to ask the question of how circular or radial a given orbit should be for either the Toth \& Ostriker (1992) derivation or ours, respectively, to apply. Clearly, given the variation in orbital parameters of the cases shown in Figure 1 and agreement of the results with our predicted quadratic dependence on $\mu$, the radial case derived here, where the imparted energy $\propto\left(M_{2} / M_{1}\right)^{2}$, is general, and the circular case in Toth \& Ostriken (1992), with imparted energy $\propto\left(M_{2} / M_{1}\right)$, is pathological. The encounters in Velazquez \& White (1999) span a range in circularity from $J / J_{C}(E)=0.3-0.8$, and the impact parameter study from Younger et al. (2008) covers $\sim 0.1-3$ in the ratio of the angular momentum to that of a circular orbit. Moreover, Hayashi \& Chiba (2006) and Ardi et al. (2003) study full orbit distributions, both isotropic and moderately radially biased, which should include large populations of initially more tangential orbits. In all cases, the end results still scale more generally with the predicted quadratic $\propto\left(M_{2} / M_{1}\right)^{2}$ rather than linear $\propto\left(M_{2} / M_{1}\right)$.

It is easy to see why this must be the case. Recall, the instantaneous work done by the secondary as it moves some distance $\mathrm{d} s$ is $\propto M_{2}^{2} \mathrm{~d} s$ (second-order in $M_{2}$ ). The case of rigid satellites on circular orbits yields a linear scaling in $M_{2}$ because the number of orbits required precisely cancels one order in $M_{2}$ - i.e. in order for the orbit to decay inward by $\mathrm{d} r$, it must execute $\sim\left(M_{1} / M_{2}\right)(\mathrm{d} r / r)$ orbits. So the net heating as it moves through the annulus is the combination of these two factors: $M_{2}^{2} \times\left(M_{1} / M_{2}\right) \sim M_{1} M_{2}$. This requires a rather precise cancellation - if any effect can upset this - i.e. if for some reason the large number of circulations is suppressed, and in fact the satellite does not execute $\sim\left(M_{1} / M_{2}\right)$ identical orbits for each unit radius it decays - then the scaling will just go as $M_{2}^{2}$.

Such a suppression is almost inevitable: first, as discussed above, a realistic extended secondary will rapidly lose mass and eventually be tidally destroyed as it executes such circulations. In practice, the secondary, especially in e.g. a 1:10 case where it would require $\sim 10$ full orbits through each radial interval to yield a linear scaling, never survives more than a couple of penetrating passages without losing a significant fraction of its mass. The subsequent passages are exponentially suppressed as the secondary loses mass, and a proper re-derivation of the heating gives an energy transfer rate that goes like $M_{2}^{2}\left[1+\exp \left(-M_{1} / M_{2}\right)\left(M_{1} / M_{2}\right)\right]-$ so the leading term is similar to our estimate for a single passage. In terms of the instantaneous heating, it does not matter whether the orbit is radial or circular - our derivation is really more broadly applicable to all cases where a very long (secondary mass-conserving) in-spiral is not possible.

Second, as discussed in $\S 2.1$, the system does not, in fact, need $\sim M_{1} / M_{2}$ circulations to lose a significant fraction of its angular momentum; N-body simulations including extended systems typically find that angular momentum exchange in resonances (and mass loss) makes only one or two close passages sufficient.

Third, in systems with a large number of bodies (halo substructure), there tends to be efficient transfer and segregation of angular momentum. The satellites on circular orbits will preferentially occupy relatively large impact-parameter orbits in the outer regions of the halo, not coming close to the disk (see e.g.Font et al.2001). The systems that will have encounters with the disk are on radial orbits, and so can be considered a series of independent highly radial passages, rather than a 
continuous circular heating. It is difficult to obtain circular orbits at small radii, and usually involves invoking slow tangential decay of the initially circular orbits at larger radii owing to dynamical friction (a slow process, with characteristic timescales of order the Hubble time).

\section{CONSEQUENCES OF THESE ORBITS}

Having established that disk heating owing to mergers with somewhat radial orbits or efficient stripping is second-order in $M_{2} / M_{1}$, whereas heating owing to mergers with rigid satellites on circular orbits is first-order in $M_{2} / M_{1}$, what are the consequences of this?

\subsection{The "Allowed" Merger History of the Milky Way and Massive Disks}

First, consider what this means for the allowed merger history of a given galaxy. Modulo the numerical prefactor, a rigid satellite on a circular orbit of mass ratio $\mu \equiv M_{2} / M_{1}$ will heat the disk to $H / R \sim \mu$. For a 1:10 merger on a circular orbit, for example, this translates to $H / R \sim 0.1$, comparable to values observed, setting a relatively stringent limit on how many mergers of even this small a mass ratio could have occurred without overheating or destroying a massive (Milky Way-like) stellar disk. However, if (more realistically) the same merger were to occur on a radial orbit and/or stripping were efficient, the heating would be suppressed by an additional factor $\sim \mu$, giving $H / R \sim \mu^{2}$. For the 1:10 merger, then, this is an orderof-magnitude suppression in the disk heating! The previous constraints in fact would allow quite a large number of high mass-ratio (or even a few fairly low mass-ratio) mergers without problems for disk survival.

Figure 2 re-examines the constraints on the Milky Way disk in light of this important distinction. Various observations (Wielen 1977; Carlberg et al. 1985; Quillen \& Garnett 2001; Nordström et al. 2004; Seabroke \& Gilmore 2007) have quantified the vertical velocity dispersion of stars in the solar neighborhood as a function of the mean stellar population age $\tau$. Given the circular velocity $V_{c} \sim 220 \mathrm{~km} \mathrm{~s}^{-1}$ and one of the models for disk heating described in $\S 2.3$ above, this can be translated to an upper limit on the allowed merger history. Note that some of the observed dispersion could owe to purely internal effects - e.g. heating by two-body relaxation and scattering off of molecular clouds, spiral structure, and other local density perturbations - so assuming that the heating comes entirely from mergers as given by the equations above is an upper limit to the allowed merger history (technically gas complicates this comparison somewhat, since it can dissipate, but as we discuss below, the effect is not large and further supports our argument that the merger history could be much more violent than has been previously assumed).

In detail, merger histories could be complex and mix many different mass ratios - however, in order to show the qualitative result in illustrative fashion, we convert each value $\sigma(\tau)$, compiled from the observations of Nordström et al. (2004), to a maximal number of 1:10 mergers assuming each merger is exactly $1: 10$, that there has been no new accretion in the meantime (again, including such a term would allow even more violent merger histories), and that each merger heats the disk according to Equation (7). We plot the resulting "maximum number of 1:10 mergers" as a function of lookback redshift corresponding to each stellar age $\tau$, assuming either radial/stripped orbits or rigid satellites on circular orbits in our conversion. We compare this to the median (and $\pm 1 \sigma$ range) number of mergers of this mass ratio or lower (more massive) integrated from $z=0$ to the corresponding lookback time $\tau$ estimated from the dark matter merger rates as a function of mass and redshift, for halo masses similar to the Milky Way $\left(\sim 10^{12} M_{\odot}\right.$, although the expectation is only weakly massdependent), from Stewart et al. (2007) and Fakhouri \& Ma (2008) (note that for a given definition of merger mass ratio, the two calculations give answers in agreement to within $\sim 20 \%$ ).

The answer is as expected from above: if the merging orbits were all circular and satellites were rigid, transfer of orbital energy to disk heating would have been efficient, and the limits are quite stringent (significantly below the expected number of mergers). On the other hand, if the mergers were radial (i.e. most of their energy went into violently relaxing and heating only the central bulge stars) and/or stripping acts efficiently after a couple of passages, then a much larger number of mergers are permitted - in fact significantly more than are cosmologically expected in typical systems (allowing some of the observed heating to stem from internal processes, and reflecting the fact that some - albeit not most - orbits will be more circular than others and yield somewhat more heating).

Similarly, we can convert the observational constraints into a maximum allowed mass ratio merger for each stellar population age (assuming the entire heating owed to a single merger, and using Equation (7) to estimate the corresponding mass ratio for either a circular/rigid or radial/non-rigid interaction). Figure 2 shows these results as well, compared to the median expected maximum mass ratio merger since lookback time $\tau$ (and $\pm 1 \sigma$ range). The dramatic qualitative difference between orbits is again present. Regardless of how we represent merger histories, the constraints on disk heating/thickness easily allow for the expected cosmological merging histories if the orbits are primarily radial and/or satellites are non-rigid (with enough cushion that even if there is internal heating and the occasional circular orbit, there is still no significant tension).

Note that while our derivation more than allows for the fraction of $>1: 10$ mergers in total, there is an intersection of our constraints with the maximum mass ratio merger around mass ratios $\sim 1: 4-1: 3$. This, of course, is expected. Roughly half of the observed galaxy population with the same mass as the Milky Way are bulge-dominated (S0 or elliptical) galaxies, with most of this mass assembled since $z \sim 1-2$ (see e.g. Bell et al. 2003; Borch et al. 2006). We would expect that the Milky Way, being still disk-dominated, would therefore be below the median (in short, the half of galaxies that have had a $\sim 1: 3$ merger since $z \sim 2$ probably correspond to the half of galaxies at these masses that are ellipticals). Allowing for this fact (or equivalently comparing the distribution of expected mergers in systems that would still be disks today), there is no tension here (the allowed history for the Milky Way is consistent with that expected for fully $\sim 1 / 3$ of galaxies of similar mass, or roughly $\sim 80 \%$ of the systems that will still be diskdominated at $z=0$ ). Furthermore, the constraints are even less stringent if we allow for gas accretion and/or subsequent growth of the galactic disk after early redshifts.

This is quite generally applicable - not just to the Milky Way disk, but to the population of observed edge-on disk galaxies. For example, Dalcanton \& Bernstein (2002) estimate the age-scale height relations for a number of observed edge-on disks of various masses. The median trends are similar to those implied by the Milky Way age-velocity dispersion 

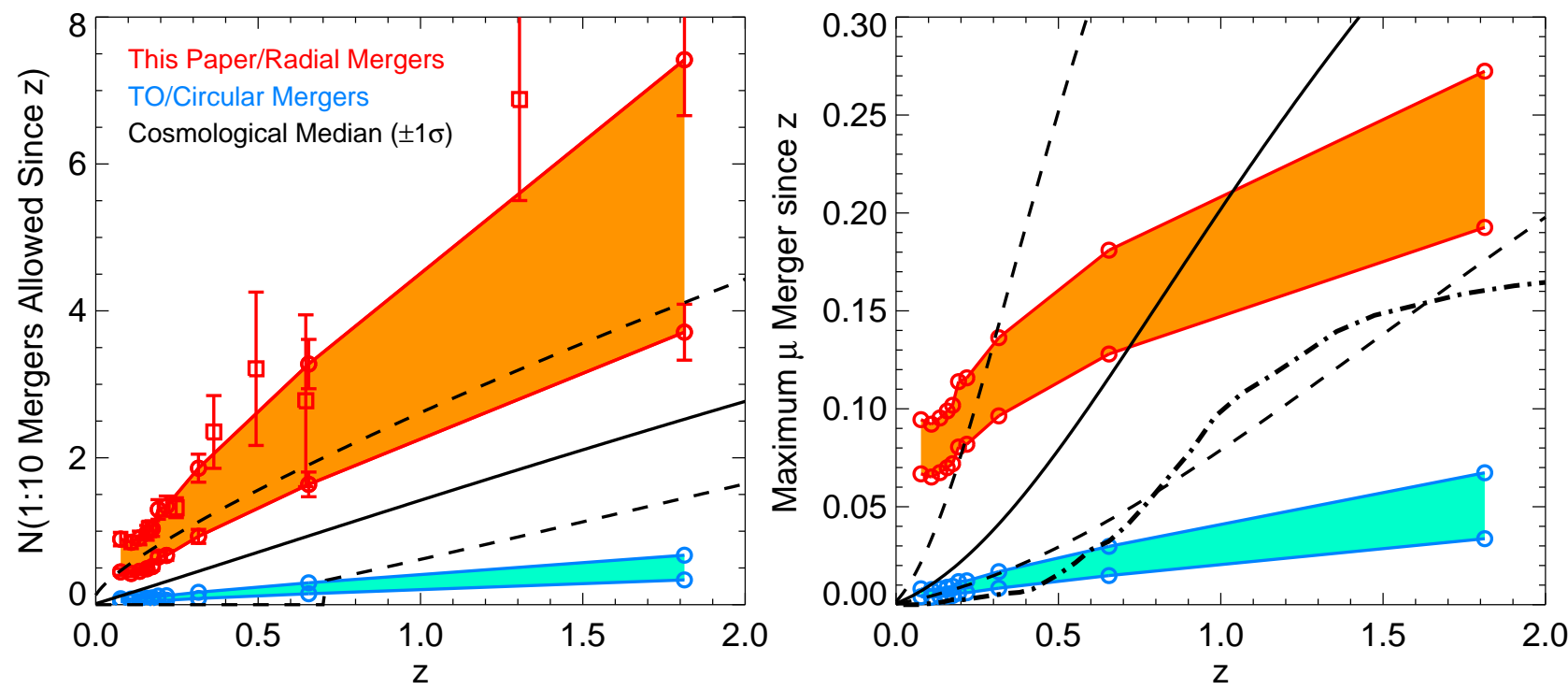

FIG. 2.- Constraints on the Milky Way merger history from the observed thick disk and local stellar age-velocity dispersion relation. Left: Number of 1:10 mergers implied by the observed age-velocity dispersion relation, since a given redshift $z$. Each point represents the constraint from observed stellar velocity dispersions in the solar neighborhood (from Nordström et al. 2004; Seabroke \& Gilmore 2007, circles and squares, respectively), at a given stellar age (here

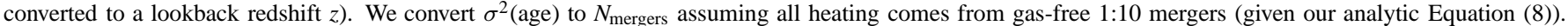
Allowing gas makes the constraints more generous by a factor $1 /\left(1-f_{\mathrm{gas}}\right)$. We contrast $N_{\text {mergers }}$ given our derivation of heating in realistic orbits (where $\Delta \sigma^{2} \propto\left(M_{2} / M_{1}\right)^{2} ;$ red $)$ and the derivation from Toth \& Ostriker (1992) for rigid satellites on circular orbits $\left(\Delta \sigma^{2} \propto M_{2} / M_{1} ;\right.$ blue). Shaded range represents the typical factor $\sim 2$ scatter in normalization (see Figure 1). We compare the median $N_{\text {mergers }}$ more major than 1:10 since redshift $z$ (solid black line; interquartile range shown as dashed lines) expected for a Milky Way-mass halo in $\Lambda$ CDM cosmological simulations (Fakhouri \& Ma 2008). Right: Same, but instead assuming that all the heating for each point was effected by a single merger - i.e. estimating the maximum allowed merger since $z$. Solid line is the cosmological expectation as before, where halo mass is defined at each redshift; dot-dashed line from Stewart et al. (2007) is the expectation where the mass ratio is relative to the $z=0$ Milky Way mass (i.e. allowing for growth of the disk since a given redshift). Observational constraints, re-analyzed with the appropriate derivations for realistic orbits, easily accommodate the expected merger histories of Milky Way-like galaxies (allowing $\sim 5-10$ individual 1:10 mergers).

relation (and in fact if we were to convert their estimates in the same manner in Figure 2, the resulting allowed ranges would lie within the same shaded range as estimated for the Milky Way, albeit with larger error bars).

\subsection{The Edge-On Shapes of Galaxies Along the Hubble Sequence}

Since both disk heating and bulge formation proceed from mergers, the differences above naturally predict a different relation between e.g. disk thickness and bulge-to-disk ratio along the Hubble sequence from very late type disks to bulgedominated systems. In Hopkins et al. (2008) we demonstrate that a mean relationship holds between the amount of classical bulge mass formed (violently relaxed and formed in a dissipational starburst owing to loss of angular momentum in the cold gaseous disks) and mass ratio, orbital parameters, and pre-merger gas content of merging galaxies. For otherwise fixed parameters at the time of merger, the amount of bulge formed scales roughly linearly in the mass ratio $\sim \mu$, for the reasons briefly discussed in $\$ 2.1$ above. Correspondingly, the amount of disk heating scales according to our estimates, as a function of merger mass ratio and gas content.

A proper quantitative calculation of e.g. the relation between bulge-to-disk ratios and disk scale heights must account for the details mentioned above - including the evolution of merger history as a function of mass ratio and the corresponding evolution in gas fractions (which affect both bulge-to-disk ratios and disk scale heights, but in somewhat different manners). Moreover, these derivations apply only to systems in a post-merger status - more properly we would need to include the effects of accretion of new gas subsequent to each event, building up disk mass and making the disk more thin. Any quantitative estimates therefore require a much more com- plete cosmological model than we have here.

However, we can highlight some of the salient qualitative behaviors that differ between our derived disk heating expectations for realistic orbits and the derivation of Toth \& Ostriker (1992), by adopting a simplified toy model. Consider a set of disks where the bulge was entirely formed by the most recent merger, of mass ratio $\mu$, and ignore subsequent accretion of new disks. The bulge mass fraction will then be $\approx \mu$, where in detail we use the formulae from Hopkins et al. (2008) for a median expected orbit (averaging over random orbits) and small pre-merger disk gas fraction $(\lesssim 10-20 \%)$.

The surviving disk will have a thickness determined by Equations (7)-(8), or the corresponding equations from Toth \& Ostriker (1992) in the strict limit of rigid satellites on circular orbits - again we assume $f_{\text {gas }} \ll 1$ for convenience and use the exact normalizations from $\S 2.3$, which agree reasonably well with full numerical experiments. We assume a disk height $H / R_{d} \approx 0.1$ for isolated systems, comparable to the expectations for purely internal heating (see e.g. Binney \& Tremaine 1987; Sellwood et al. 1998) and isolated disk galaxy simulations (Villalobos \& Helmi 2008; Younger et al. 2008). These details are important only for the exact numerical quantities in the profiles - the behavior of interest is that, for the cases we derive here, we roughly obtain $H / R \sim(B / T)^{2}$ (plus some constant; since $\Delta H / R \propto \mu^{2}$ and $B / T \propto \mu)$, whereas for the Toth \& Ostriker (1992) case we have $H / R \sim(B / T)$ (both $\propto \mu)$. The qualitative relationship between $H / R$ and $B / T$ is different.

Assuming the initial disk is Milky Way-like (giving it the appropriate size and mass), and giving the bulge the appropriate projected effective radius from the spheroid size-mass relation in Shen et al. (2003) for its mass (knowing B/T), we can then construct a mock two-dimensional profile of the system. 


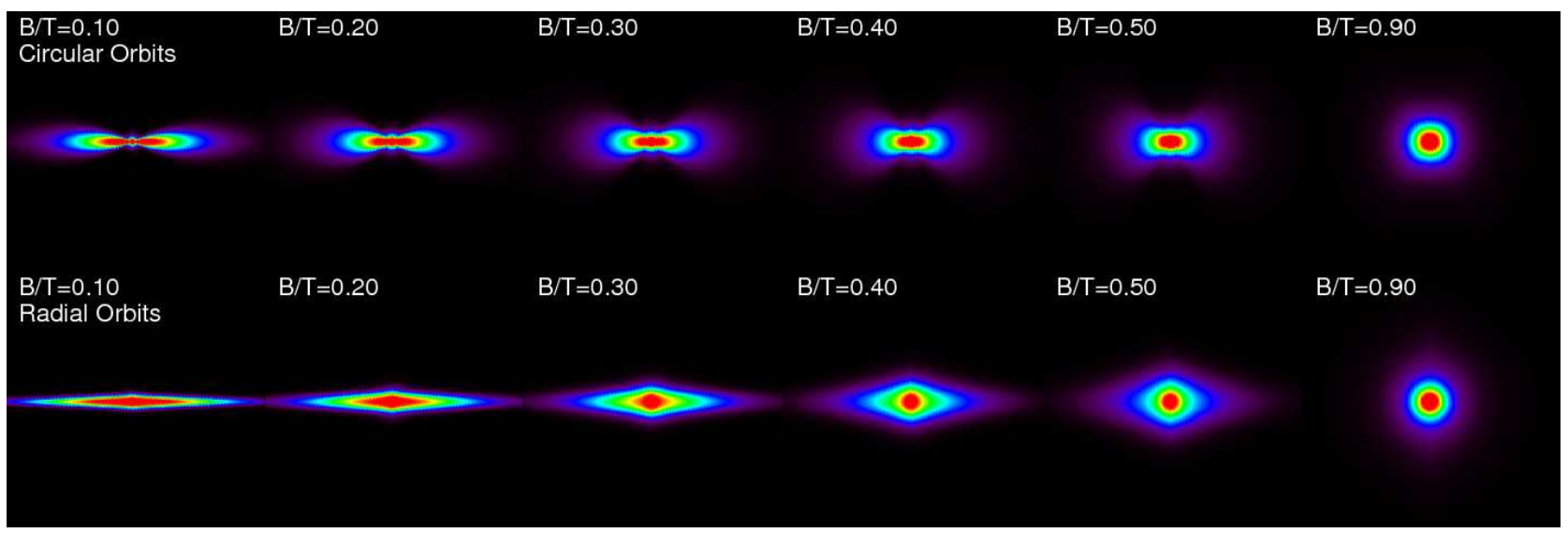

FIG. 3.- Qualitative (toy model) expectations for the difference in edge-on morphologies of disk+bulge systems (with the given bulge-to-total stellar mass ratio $B / T$ ) given disk heating according to our derivation for realistic orbits (bottom) and that for rigid satellites on circular obits (top). In the toy model, the bulge fraction traces the mass ratio of the most recent (assumed gas-poor) merger, according to the calibration from numerical experiments in Hopkins et al. (2008); we then calculate the amount of disk heating given this merger, and plot the combined, projected edge-on surface brightness profile of the system. Because disk heating is much more efficient in the rigid satellite, circular orbit regime, disks in intermediate $B / T$ systems are much thicker than observed - if heights scaled as predicted they would "swamp" the bulge and give rise not to a bulge plus disk morphology but to a series of decreasingly elliptical "boxy" (rectangular) isophotes. In contrast, our prediction for relatively inefficient heating means the disks even in intermediate $B / T$ systems can be relatively thin $(H / R \lesssim 0.2)$, leading to a standard bulge plus disk edge-on morphology and the characteristic observed "disky" isophotes in large bulges with embedded disks.

For simplicity, assume that the "bulge" is spherically symmetric with a projected de Vaucouleurs (1948) profile and the disk is azimuthally symmetric with an exponential surface brightness profile and vertical $\operatorname{sech}^{2}$ profile (with the estimated $H / R)$. Note that it makes no difference to our qualitative comparisons whether we adopt e.g. a pure exponential vertical profile instead, as suggested by Barteldrees \& Dettmar (1994); de Grijs et al. (1997). Integrating through these mock profiles, we can construct the edge-on "surface brightness" map of the total system, and show this in Figure 3 . For both our estimate of disk heating here, and that in Toth \& Ostriker (1992), we construct this toy model image for cases with net values $B / T=0.1-1$.

The difference in galaxy shapes is immediately apparent. Again, the quantitative details will, in reality, reflect a much more complex history of the system. Here, we focus on two qualitative details. First, if heating were as efficient as the derivation of Toth \& Ostriker (1992) implies, there are essentially no very thin disks, even in systems with low $B / T$. This is similar to what we have found in $\$ 3.1$ above - the amount of heating for some merger history is, for realistic orbits, much less. Second, at intermediate $B / T$ typical of early-type spirals and S0 galaxies, the derivation herein implies (owing to the low efficiency of disk heating) that disks, while clearly thicker, are still thinner than the bulge itself, giving galaxies a reasonably defined disk plus bulge or disk plus spheroid edgeon morphology, comparable to what is canonically observed along the Hubble sequence. On the other hand, if heating were linear in $\mu$, then it would more or less track $B / T$, giving thick disks (for $B / T \sim 0.3-0.5$, this gives disk $H / R \sim 0.3-0.5$, comparable to what is typically associated with galaxy bulges and extended spheroids, not "disks" in normal observational parlance). In fact, this is sufficient to largely suppress the "disk plus bulge" morphology entirely - the Hubble sequence and changing bulge to disk ratios do not appear as such when viewed edge on, but rather (visually) would appear to be primarily a sequence in disk thickness.

\section{DISCUSSION AND CONCLUSIONS}

We have shown that allowing for realistic satellite orbits in particular the fact that orbits are not exactly circular, and that satellites tend to be increasingly stripped as they execute multiple passages through a disk - has dramatic implications for the efficiency and cosmological evolution of disk "heating" via minor mergers and galaxy-galaxy interactions.

In previous generations of analyses (e.g. Toth \& Ostriker 1992), several assumptions were made implicitly: that a satellite would enter a disk on a slowly-decaying circular orbit owing to dynamical friction, and that the satellite was rigid. In addition, much of this work focused on dissipationless (gasfree) cases. Under these conditions, it is straightforward to derive the fractional disk heating owing to a merger of a satellite of mass $M_{2}$ with a primary of mass $M_{1}$, and to show that this heating (and correspondingly the induced increase in disk scale height and vertical velocity dispersion) scale linearly in the mass ratio, $\propto M_{2} / M_{1}$.

This fundamental premise has largely informed the literature on the subject of disk heating and empirical constraints on merger histories from the thickness and vertical profiles of observed disks. However, we show that it is no longer the case when the simplifying assumptions are relaxed (as in current, more detailed simulations and cosmological models; e.g. Velazquez \& White 1999; Ardi et al. 2003; Benson et al. 2004; Hayashi \& Chiba 2006; Kazantzidis et al. 2007). These assumptions are fine when one considers a system that is effectively a point particle at large radius in an extended (but relatively low-density) background halo, on a moderately circular orbit, such that there are no close passages of the satellite and primary and the entire regime of the orbit has a characteristic frequency much larger than the internal characteristic orbital frequency of either the primary or secondary - in the regime where the satellite is far from the galaxy and has just been accreted near the halo virial radius, for example. And in a strictly instantaneous sense, they are not bad. But in a global sense, in terms of estimating the effects on a galactic disk from a merger with initial mass ratio $M_{1} / M_{2}$, these assumptions break down, and the result is not just a numerical correction, but a qualitative change in the character of the heating.

In modern $\Lambda \mathrm{CDM}$ cosmologies, orbits tend to be highly radial, especially those orbits that actually pass near or through the disk. Circular orbits efficiently lose angular momentum 
to interactions and other substructures in halo formation, and even when they survive, the dynamical friction times required for the orbit to decay from near the virial radius are so large (of order the Hubble time) that they tend to survive far away from the central galaxy rather than merge with and/or heat the disk. Moreover, once the galaxy gets sufficiently close to the disk that it might effect some heating, the characteristic orbital frequencies are (by necessity) similar to the characteristic internal frequencies of the disk and halo. Because some of the galaxy will be in near-resonance with the secondary orbit, this allows much more efficient transfer of angular momentum from the orbital motion into internal motions, and even initially circular orbits become radial in just one or two passages. Because the orbital angular momentum is transferred quickly in a passage, the secondary cannot gently "spiral in" but must return from apocenter in a radial "plunge" - the secondary effectively free-falls inwards (weakly decelerated by e.g. dynamical friction at this point) and "punches through" the primary, where it executes a rapidly damping oscillation through the primary on nearly radial orbits. At this stage, violent relaxation scatters stars off the time-dependent potential and transfers the orbital energy to the central stellar distribution (the bulge or whatever the hottest stars are at the center of the galaxy), damping the orbit and completing coalescence in a couple of dynamical times. At the same time, once the secondary begins to penetrate the primary disk, it experiences rapid tidal mass loss and soon most of the mass has been tidally added to the extended halo and cannot further heat the disk.

For these reasons, the most useful approximation for disk heating in realistic cosmological situations is not a gradually decaying circular orbit of a rigid satellite, but rather something like the impact approximation: the heating is dominated by a single or a couple of radial oscillations at the initial mass, on a radial free-fall trajectory. We show that in this case, the heating is not linear in the mass ratio $M_{2} / M_{1}$. Rather, the effective heating is second-order in the mass ratio, $\propto\left(M_{2} / M_{1}\right)^{2}$. We show that - again for the reasons above - this is a much more robust scaling.

We demonstrate this by comparing our analytic predictions to a variety of numerical simulations, which include a range in secondary mass ratios, assumed structural properties of the disk, halo, bulge, and satellite, gas content, redshift, orbital parameters, and orbital anisotropy and angular momentum content. Remarkably, these different simulations, from various independent groups, all agree with our analytic predicted scalings and with each other (when properly normalized) to within a factor $\sim 2-3$. It is straightforward to show that a linear scaling in mass ratio is not a good description of the simulation results (even if the normalization of the linear scaling is free - i.e. the scaling itself must be second-order).

This provides a physical reason why simulations increasingly find, contrary to historical expectations, that systems can undergo cosmologically expected merger histories and still yield relatively thin disks at $z=0$ in line with observations (see e.g. Velazquez \& White 1999; Ardi et al. 2003; Abadi et al.|2003; Benson et al.|2004; Robertson et al. 2004, 2006; Okamoto et al. 2005; Havashi \& Chiba 2006; Governato et al.|2007; Kazantzidis et al.|2007; Younger et al. 2008; Villalobos \& Helmi 2008). Many of these studies found that idealized numerical experiments yield less efficient disk heating than that predicted by Toth \& Ostriker (1992); the key contribution of our work is to outline the physical reasons for this difference and to demonstrate that these differences are not a matter of e.g. the normalization or numerical prefactors involved in an estimate of disk heating: heating is secondorder in mass ratio $(\mu)$.

This qualitatively changes the disk heating paradigm. Because the mass spectrum of mergers (in terms of halo mass) goes as $\mathrm{d} N / \mathrm{d} \ln \mu \propto \mu^{-1}$, if the heating per encounter were in fact linear $(\propto \mu)$, then there would be more or less equal contributions to disk heating and thick disk formation from each logarithmic interval in mass ratio. Observationally, this would imply that no more than a few dwarf galaxies with properties similar to those of e.g. the rather small Sagittarius dwarf (see Helmi 2004; Fellhauer et al. 2006) could have been accreted or destroyed by the Milky Way in the last $10 \mathrm{Gyr}$, in conflict with cosmological expectations and making the well-known "missing satellite" problem even more difficult to resolve.

In contrast, if the heating is second-order in $\mu$, then the net heating is dominated by the few large mergers of relatively large mass ratios. This behavior has in fact been recognized in cosmological simulations, and yields better agreement with observations (see Abadi et al. 2003; Robertson et al. 2004; Benson et al. 2004; Okamoto et al. 2005; Governato et al. 2007; Kazantzidis et al. 2007; Read et al. 2008). It also fundamentally enables the existence of very thin disks: since mergers of relatively large mass ratios $\mu \gtrsim 0.1$ are rare, they are somewhat stochastic - i.e. some systems which have gone sufficient time or been sufficiently gas rich since their last $\sim 1: 10$ merger will exist and can therefore have very thin disks $(H / R \lesssim 0.1)$ similar to the "ultra-thin" or "superthin" disks observed (Seth et al. 2005; ; Yoachim \& Dalcanton 2006). If instead the same net heating came from mergers of mass ratios $\mu \sim 0.001-0.01$ (where the expected number of mergers since $z \sim 1$ is of order several hundred per galaxy; making the existence of any galaxies without such mergers in their recent past an improbable event) as from mergers with mass ratio $\mu \sim 0.1-1$, then it would be almost impossible to contrive realistic cosmological merger histories that could give rise to ultra-thin disks.

There are a number of other indirect implications of this result. For example, we demonstrate that this is of significant importance for the edge-on morphologies of disk plus bulge systems. Given some bulge formation in mergers, whether the induced heating in the surviving disk goes linearly or second-order in $\mu$ produces a different relation between scale heights and bulge-to-disk ratios and, correspondingly, different morphologies in observed early-type disks. If realistic scale heights increased as efficiently as expected in the circular orbit, rigid secondary approximation, then disks of intermediate $B / T$ would necessarily be thicker than observed - in fact, the Hubble sequence, viewed edge-on, would no longer have a characteristic "bulge plus disk" morphology and would instead appear as a sequence in ellipticity of characteristically boxy isophotal shapes (because a sufficiently thick disk appears in edge-on projection as somewhat rectangular). In contrast, disks appear to remain thin even in systems with significant (or dominant) bulges, giving rise to characteristically "disky" ("lemon-shaped") isophotal shapes. The nonlinearity of disk heating can explain why galactic morphological components form bimodal families (bulges and disks), as opposed to a sequence of increasingly "spheroidal" disks.

Applying these recalibrated models to the Milky Way and other massive disk galaxies, the implied constraints on the merger history from the thick and thin disk are dramatically 
changed and fully consistent with cosmologically expected merger histories. In detail, applying our scaling, calibrated against numerical simulations, to the observed age-velocity dispersion relation in the solar neighborhood, implies that the Milky Way could have survived as many as $\sim 5-10$ minor (mass ratio $\sim 1: 10$ ) mergers in the last $10 \mathrm{Gyr}$. If some of the disk thickness owes to other processes or there were some more violent events, this number might be a bit smaller, but if the Milky Way were more gas-rich in the past, then this number would in fact go up by a factor $1 /\left(1-f_{\text {gas }}\right)$. In either case, the median number of such mergers expected for a MilkyWay mass halo is only $\sim 3$ (with $\sim 90 \%$ of Milky-Way like systems having $<5-6$ such mergers in this interval). The observed vertical velocity dispersions of stars (and implied disk thickness) of the Milky Way is completely consistent with the cosmologically expected amount of minor merging in the last $10 \mathrm{Gyr}$, and in fact these constraints alone permit the Milky Way, far from being especially quiescent, to be in the upper cosmological quartile in terms of number of minor mergers since $z \sim 1-2$. Translating these constraints to a "maximum" mass ratio merger implies that the Milky Way could even have survived mergers of mass ratio $\sim 1: 4-1: 3$ since $z \sim 2$, without producing too much heating or thick disk.

Because the heating is second order in mass ratio, there is almost no measurable heating effected by encounters with mass ratios $\mu \ll 0.1$, so there is no overall constraint based on the thickness of the disk or internal velocity dispersions on how much total mass has been added to the Milky Way via merging. In contrast to the $<5 \%$ growth since $z \sim 2$ inferred by Toth \& Ostriker (1992), we find that the Milky Way could have accumulated up to $\sim 25-50 \%$ of its present mass in the last 10 Gyr by $1: 10$ mergers alone $(\sim 5-10$ individual $1: 10$ mergers). More interesting, because again smaller mass ratio mergers heat only to second order in $\mu$, the Milky Way could easily, in fact, have grown by factors of several since $z \sim 2$, provided this growth mostly came from relatively small mass ratio mergers $\mu \lesssim 1: 10$. This is critical for our understanding of galaxy formation: at some level of sufficiently small mass ratios below $\sim 1: 10-1: 100$, the distinction between accretion and mergers is increasingly blurred. Essentially all galaxies are expected to gain a large fraction of their mass and grow by large factors (up to an order of magnitude) in the last $\sim 10 \mathrm{Gyr}$ from such accretion events. If these small events heated as efficiently as major events (e.g. if $\sim 30$ separate 1:100 mergers were as damaging to a disk as a 1:3 merger), there would be a fundamental difficulty for CDM models. In fact, owing to the inefficiency of disk heating, there is no tension here.

This is not, of course, to say that problems of disk for- mation and survival in a $\Lambda$ CDM Universe have been solved. There are still well-known problems related to the loss of angular momentum in disks (owing in part to over-merging), that tend to yield (in cosmological simulations) disks that are too concentrated and have too much bulge relative to observations. A great deal of theoretical effort has gone into studying this (Weil et al. 1998; Sommer-Larsen et al. 1999, 2003; Thacker \& Couchman 2000, 2001; Abadi et al. 2003; Governato et al. 2004, 2007; Robertson et al. 2004; Okamoto et al. 2005; (Scannapieco et al. 2008), with increasing agreement that some combination of improved resolution and proper implementation of feedback from supernovae and stellar winds are critical for the formation of realistic disks. These issues are not addressed or affected by our analysis here.

However, it is increasingly clear (as resolution improves such that thick disks can be properly resolved in the vertical direction) that these simulations do not generally have the problem of producing disks that are much too thick relative to observations. In this paper, we have shown that there is a good physical reason for this, and that it is not a peculiarity of a couple of simulations or of an especially quiescent merger history, but rather is a robust and quite general expectation even for systems with violent merger histories and even in systems that will be bulge-dominated at $z=0$. We have derived a new, more generally applicable scaling of disk height with merger mass ratio, and shown that it qualitatively changes the expectations for how disk heating should proceed in a $\Lambda \mathrm{CDM}$ universe, and generically leads to the expectation that disks are quite robust to being heated by minor mergers. Although there are still considerable improvements in models needed to explain when and how disks form and why the distribution of bulge-to-disk ratios is what is observed at $z=0$, there is no tension between the models and the heating and thickness of disks. In short, we have argued that there is no "disk heating" problem in $\Lambda \mathrm{CDM}$ cosmologies, once a proper accounting of disk heating for realistic cosmological orbits and conditions is considered.

We thank Shardha Jogee, John Kormendy, and Todd Thompson for helpful discussions. We also thank Kyle Stewart for sharing suggestions and simulation results. This work was supported in part by NSF grants ACI 96-19019, AST 0071019, AST 02-06299, and AST 03-07690, and NASA ATP grants NAG5-12140, NAG5-13292, and NAG5-13381. Support for TJC was provided by the W. M. Keck Foundation.

\section{REFERENCES}

Abadi, M. G., Navarro, J. F., \& Steinmetz, M. 2006, MNRAS, 365, 747

Abadi, M. G., Navarro, J. F., Steinmetz, M., \& Eke, V. R. 2003, ApJ, 597, 21

Ardi, E., Tsuchiya, T., \& Burkert, A. 2003, ApJ, 596, 204

Barnes, J. E. 1988, ApJ, 331, 699

-. 1992, ApJ, 393, 484

Barnes, J. E., \& Hernquist, L. 1996, ApJ, 471, 115

Barnes, J. E., \& Hernquist, L. E. 1991, ApJ, 370, L65

Barteldrees, A., \& Dettmar, R.-J. 1994, A\&AS, 103, 475

Barton, E. J., Arnold, J. A., Zentner, A. R., Bullock, J. S., \& Wechsler, R. H 2007, ApJ, 671, 1538

Bell, E. F., McIntosh, D. H., Katz, N., \& Weinberg, M. D. 2003, ApJS, 149, 289

Bell, E. F., Phleps, S., Somerville, R. S., Wolf, C., Borch, A., \& Meisenheimer, K. 2006, ApJ, 652, 270

Benson, A. J. 2005, MNRAS, 358, 551
Benson, A. J., Lacey, C. G., Frenk, C. S., Baugh, C. M., \& Cole, S. 2004, MNRAS, 351, 1215

Besla, G., Kallivayalil, N., Hernquist, L., Robertson, B., Cox, T. J., van der Marel, R. P., \& Alcock, C. 2007, ApJ, 668, 949

Binney, J., \& Tremaine, S. 1987, Galactic dynamics (Princeton, NJ, Princeton University Press, 1987)

Bizyaev, D., \& Mitronova, S. 2002, A\&A, 389, 795

Borch, A., et al. 2006, A\&A, 453, 869

Boylan-Kolchin, M., Ma, C.-P., \& Quataert, E. 2008, MNRAS, 383, 93

Bridge, C. R., et al. 2007, ApJ, 659, 931

Carlberg, R. G., Dawson, P. C., Hsu, T., \& Vandenberg, D. A. 1985, ApJ, 294, 674

Cole, S., \& Lacey, C. 1996, MNRAS, 281, 716

Dalcanton, J. J., \& Bernstein, R. A. 2002, AJ, 124, 1328

de Grijs, R., \& Peletier, R. F. 1997, A\&A, 320, L21

de Grijs, R., Peletier, R. F., \& van der Kruit, P. C. 1997, A\&A, 327, 966 
de Vaucouleurs, G. 1948, Annales d'Astrophysique, 11, 247

Fakhouri, O., \& Ma, C.-P. 2008, MNRAS, 386, 577

Fellhauer, M., et al. 2006, ApJ, 651, 167

Font, A. S., Navarro, J. F., Stadel, J., \& Quinn, T. 2001, ApJ, 563, L1

Gilmore, G., Wyse, R. F. G., \& Norris, J. E. 2002, ApJ, 574, L39

Governato, F., Willman, B., Mayer, L., Brooks, A., Stinson, G., Valenzuela,

O., Wadsley, J., \& Quinn, T. 2007, MNRAS, 374, 1479

Governato, F., et al. 2004, ApJ, 607, 688

Hashimoto, Y., Funato, Y., \& Makino, J. 2003, ApJ, 582, 196

Hayashi, H., \& Chiba, M. 2006, PASJ, 58, 835

Helmi, A. 2004, ApJ, 610, L97

Hernquist, L. 1989, Nature, 340, 687

-. 1990, ApJ, 356, 359

-. 1992, ApJ, 400, 460

-. 1993, ApJ, 409, 548

Hernquist, L., \& Mihos, J. C. 1995, ApJ, 448, 41

Hopkins, P. F., Cox, T. J., Younger, J. D., \& Hernquist, L. 2008, ApJ, submitted, arXiv:0806.1739 [astro-ph], 806

Howley, K. M., Geha, M., Guhathakurta, P., Montgomery, R. M., Laughlin, G., \& Johnston, K. V. 2008, ApJ, in press arXiv:0804.0798 [astro-ph], 804

Kallivayalil, N., van der Marel, R. P., \& Alcock, C. 2006a, ApJ, 652, 1213

Kallivayalil, N., van der Marel, R. P., Alcock, C., Axelrod, T., Cook, K. H., Drake, A. J., \& Geha, M. 2006b, ApJ, 638, 772

Kartaltepe, J. S., et al. 2007, ApJS, 172, 320

Kazantzidis, S., Bullock, J. S., Zentner, A. R., Kravtsov, A. V., \& Moustakas, L. A. 2007, ApJ, in press, arXiv:0708.1949 [astro-ph], 708

Khochfar, S., \& Burkert, A. 2006, A\&A, 445, 403

King, I. R. 1966, AJ, 71, 64

Lin, L., et al. 2004, ApJ, 617, L9

-. 2008, ApJ, in press, arXiv:0802.3004 [astro-ph], 802

Lotz, J. M., et al. 2008, ApJ, 672, 177

Maller, A. H., Katz, N., Kereš, D., Davé, R., \& Weinberg, D. H. 2006, ApJ, 647, 763

Mateo, M., Olszewski, E. W., Vogt, S. S., \& Keane, M. J. 1998, AJ, 116 , 2315

Mestel, L. 1963, MNRAS, 126, 553

Mo, H. J., Mao, S., \& White, S. D. M. 1998, MNRAS, 295, 319

Muñoz, R. R., Majewski, S. R., \& Johnston, K. V. 2008, ApJ, 679, 346

Naab, T., \& Burkert, A. 2003, ApJ, 597, 893

Nordström, B., Mayor, M., Andersen, J., Holmberg, J., Pont, F., Jørgensen, B. R., Olsen, E. H., Udry, S., \& Mowlavi, N. 2004, A\&A, 418, 989

Okamoto, T., Eke, V. R., Frenk, C. S., \& Jenkins, A. 2005, MNRAS, 363 , 1299

Ostriker, J. P., \& Tremaine, S. D. 1975, ApJ, 202, L113

Piatek, S., Pryor, C., \& Olszewski, E. W. 2008, AJ, 135, 1024

Quillen, A. C., \& Garnett, D. R. 2001, in Astronomical Society of the Pacific Conference Series, Vol. 230, Galaxy Disks and Disk Galaxies, ed. J. G. Funes \& E. M. Corsini, 87-88
Quinn, P. J. 1984, ApJ, 279, 596

Quinn, P. J., \& Goodman, J. 1986, ApJ, 309, 472

Quinn, P. J., Hernquist, L., \& Fullagar, D. P. 1993, ApJ, 403, 74

Read, J. I., Lake, G., Agertz, O., \& Debattista, V. P. 2008, MNRAS, in press arXiv:0803.2714 [astro-ph], 803

Robertson, B., Bullock, J. S., Cox, T. J., Di Matteo, T., Hernquist, L., Springel, V., \& Yoshida, N. 2006, ApJ, 645, 986

Robertson, B., Yoshida, N., Springel, V., \& Hernquist, L. 2004, ApJ, 606, 32 Scannapieco, C., Tissera, P. B., White, S. D. M., \& Springel, V. 2008,

MNRAS, in press arXiv:0804.3795 [astro-ph], 804

Seabroke, G. M., \& Gilmore, G. 2007, MNRAS, 380, 1348

Sellwood, J. A., Nelson, R. W., \& Tremaine, S. 1998, ApJ, 506, 590

Seth, A. C., Dalcanton, J. J., \& de Jong, R. S. 2005, AJ, 130, 1574

Shen, S., Mo, H. J., White, S. D. M., Blanton, M. R., Kauffmann, G., Voges,

W., Brinkmann, J., \& Csabai, I. 2003, MNRAS, 343, 978

Sohn, S. T., et al. 2007, ApJ, 663, 960

Somerville, R. S., et al. 2008, ApJ, 672, 776

Sommer-Larsen, J., Gelato, S., \& Vedel, H. 1999, ApJ, 519, 501

Sommer-Larsen, J., Götz, M., \& Portinari, L. 2003, ApJ, 596, 47

Stewart, K. R., Bullock, J. S., Wechsler, R. H., Maller, A. H., \& Zentner,

A. R. 2007, ApJ, in press arXiv:0711.5027 [astro-ph], 711

Thacker, R. J., \& Couchman, H. M. P. 2000, ApJ, 545, 728

-. 2001, ApJ, 555, L17

Toomre, A. 1977, in Evolution of Galaxies and Stellar Populations, ed.

B. M. Tinsley \& R. B. Larson, 401

Toomre, A., \& Toomre, J. 1972, ApJ, 178, 623

Toth, G., \& Ostriker, J. P. 1992, ApJ, 389, 5

van den Bosch, F. C., Lewis, G. F., Lake, G., \& Stadel, J. 1999, ApJ, 515, 50

Velazquez, H., \& White, S. D. M. 1999, MNRAS, 304, 254

Villalobos, Á., \& Helmi, A. 2008, MNRAS, in press arXiv:0803.2323 [astro-ph], 803

Walker, I. R., Mihos, J. C., \& Hernquist, L. 1996, ApJ, 460, 121

Weil, M. L., Eke, V. R., \& Efstathiou, G. 1998, MNRAS, 300, 773

White, S. D. M., \& Rees, M. J. 1978, MNRAS, 183, 341

Wielen, R. 1977, A\&A, 60, 263

Woods, D. F., \& Geller, M. J. 2007, AJ, 134, 527

Woods, D. F., Geller, M. J., \& Barton, E. J. 2006, AJ, 132, 197

Wyse, R. F. G., Gilmore, G., Norris, J. E., Wilkinson, M. I., Kleyna, J. T.,

Koch, A., Evans, N. W., \& Grebel, E. K. 2006, ApJ, 639, L13

Yoachim, P., \& Dalcanton, J. J. 2006, AJ, 131, 226

Younger, J. D., Hopkins, P. F., Cox, T. J., \& Hernquist, L. 2008, ApJ,

submitted, arXiv:0804.2672 [astro-ph], 804 